\title{
Reforma Psiquiátrica, luta antimanicomial e novas formas de institucionalização
}

\author{
Psychiatric reform, antimanicomial struggle and new forms of institutionalization
}

Fernanda de Lima Fontelles ${ }^{\dagger}$, Roberta Barbosa da Silva

Como citar esse artigo. Fontelles, F.L; da Silva, R.B. Reforma Psiquiátrica, luta antimanicomial e novas formas de institucionalização. Revista Mosaico. 2019 Jul/Dez;; 10 (2):Suplemento 124131

\author{
Resumo \\ O presente artigo tem como propósito analisar comparativamente, através de uma revisão bibliográfica, os objetivos da reforma \\ psiquiátrica e da luta antimanicomial e o panorama atual. Assim, considerando os diversos avanços já obtidos, apresenta como \\ contribuição o apontamento de possíveis retrocessos e áreas ainda não reformadas, além de novas formas de institucionalização. \\ Entre os temas explorados estão a história da reforma psiquiátrica, o processo de desinstitucionalização e os desdobramentos \\ recentes. Conclui-se que o processo de reforma psiquiátrica ocorre de maneira contínua, apesar de já apresentar uma melhora \\ significativa no cuidado em saúde mental, sendo de suma importância para a garantia dos direitos humanos e da inclusão. \\ Palavras-chave: Reforma psiquiátrica, Luta antimanicomial, Desinstitucionalização, Medicalização.
}

\begin{abstract}
The purpose of this article is to comparatively analyze, through a literature review, the objectives of the psychiatric reform and the antimanicomial struggle and the current panorama. Thus, considering these veral advances already made, it presents as a contribution the indication of possible set back sand areas still notreformed, as well as new forms of institutionalization. Among the themes explored there are the psychiatric reform history, the deinstitutionalization process and recent developments. It is concluded that the process of psychiatric reform occurs continuously, although it already presents a significant improvement in mental healthcare, being of short importance for the guarantee of human rights and inclusion.

Keywords: Psychiatric reform, Antimanicomial struggle, Deinstitutionalization, Medicalization.
\end{abstract}

\section{Introdução}

O processo de reforma psiquiátrica e de luta antimanicomial foram importantes processos no contexto da psicologia no Brasil. Apesar de estarem em constante evolução, são notórias as mudanças já ocorridas. Dessa forma, entre os temas a serem discutidos, o artigo apresenta um levantamento acerca história da reforma psiquiátrica, suas reinvindicações e soluções propostas; as práticas atuais - com destaque para o processo de desinstitucionalização e suas consequências, tais como a medicalização e, por fim, os desdobramentos recentes.

São passíveis de observação os avanços e retrocessos advindos da reforma psiquiátrica e da luta antimanicomial desde seus primórdios, sendo possível pensar acerca das práticas atuais, levando a um questionamento das mesmas.

Partindo da temática da reforma psiquiátrica e da luta antimanicomial, o presente artigo possui o objetivo geral de problematizar a possível existência de novas formas de institucionalização e como as mesmas se mantém, pensando na produção da loucura e como ela é encarada atualmente, além de também analisar os meios com os quais se lidam com ela e sua eficácia.

O artigo se dá na forma de revisão de literatura, partindo de um levantamento de estudos anteriores buscando identificar nas publicações existentes, os avanços e retrocessos dessas lutas como forma de comparação entre os objetivos propostos inicialmente e os que foram de fato alcançados. As publicações anteriores que serviram de base ao artigo foram localizadas através dos bancos de dados online Scielo e Bireme, onde foram utilizadas as seguintes palavraschaves:"desinstitucionalização"; "reformapsiquiátrica"; "luta antimanicomial", e "medicalização".

É importante destacar que o presente artigo busca reafirmar o caráter permanente e necessários dessas lutas, não como uma crítica, mas como uma análise, afirmando-se que qualquer tipo de retrocesso deve ser

Afiliação dos autores: †Graduanda, Curso de Psicologia, Universidade de Vassouras, Vassouras, RJ, Brasil.

¥Mestre em Psicologia, Professora do Curso de Psicologia, Universidade de Vassouras, Vassouras, RJ, Brasil.

* Email de correspondencia: fernanda fontelles@yahoo.com.br 
combatido por toda a classe trabalhadora em saúde mental.

\section{O surgimento da reforma psiquiátrica e seus desdobramentos:}

No Brasil, a reforma psiquiátrica teve início em meados dos anos 70 e segundo Tenório (2002), teve como característica marcante a reinvindicação da cidadania do louco, considerando a elaboração de propostas de transformação no tratamento em saúde mental, e se desdobrou na diversificação das práticas e saberes. Joel Birman e Jurandir Freire Costa (1994), apontam que a noção de saúde mental se refere a uma boa adaptação ao social.

Este movimento de reforma psiquiátrica se iniciou a partir de denúncias de maus tratos e abandono, que voltaram a atenção para a forma como a loucura era tratada, assim, começaram a surgir críticas e questionamentos que levaram a repensar sobre as práticas. Contudo, não se tratava mais de melhorar as estruturas existentes, e sim criar novos dispositivos e tecnologias de cuidado.

A tarefa da reforma psiquiátrica, segundo Tenório (2002, p.35) era: "substituir uma psiquiatria centrada no hospital por uma psiquiatria sustentada em dispositivos diversificados, abertos e de natureza comunitária ou territorial".

Além de um grande marco na assistência em saúde mental, a reforma psiquiátrica é também um importante movimento político e social. De acordo com Bueno (2009), ela busca romper com a lógica tutelar e excludente, colocando o louco como sujeito de direitos, onde dentre seus princípios éticos estão a inclusão, a solidariedade e a cidadania.

No campo político, a reforma objetiva por ações micro e macro políticas, juntamente com os movimentos sociais, de forma a consolidar a Política Nacional de Saúde Mental (PNSM), dessa forma, de acordo com Macedo e Dimenstein (2013), é importante afirmar que a inserção de psicólogos nas políticas públicas, especialmente em saúde mental, é um forte dispositivo para problematizações acerca da atuação, identidade profissional e compromisso social dos mesmos. É necessário que tais âmbitos - político e social -, sejam destacados ao se falar em reforma psiquiátrica, visto que seu andamento - um processo contínuo - está vinculado às políticas sociais, sendo, de certa forma, dependente delas.

A criação dos Centros de Atenção Psicossocial (CAPS) e Núcleos de Atenção Psicossocial (NAPS), através da portaria GM 224/92, foi um dos marcos da história da reforma, pois, apesar de não dispensarem o saber da psiquiatria, apresentavam uma nova forma de tratamento da doença mental. Juntamente com a criação de novas redes, foram instituídas regras mais rígidas no funcionamento dos hospitais que atendiam os doentes mentais e a progressiva diminuição dos leitos, que, para a psiquiatria, é um índice positivo, visto que acompanha a criação de alternativas de cuidado. Tenório (2002) afirma que a reforma tem alcançado os objetivos propostos, porém, que ainda há muito a ser feito.

Para realizar uma efetiva mudança no tratamento e relação com a loucura, é preciso investir em uma boa rede de cuidados e também modificar a atitude frente à loucura, de forma que possa haver realmente uma integração à sociedade.

\section{O processo de desinstitucionalização: o fim dos manicômios?}

De acordo com Venturini (2010), inicialmente, o foco da desinstitucionalização foi centrado na superação dos manicômios, se estendendo posteriormente para outras práticas que seguiam o mesmo processo. Para o autor, o termo se refere ao período onde as políticas sociais e as intervenções legislativas geraram como consequência uma crise na centralidade do tratamento no manicômio, representando um grande processo de reforma.

Acerca do processo de desinstitucionalização, Amarante (2003) alerta para o risco de um processo de capsização da reforma psiquiátrica, ao apontar que a política nacional de saúde mental está reduzida a implantação de novos CAPS, e não ao processo social complexo que deve ser característica da reforma. Contudo, é preciso cautela ao considerar tal afirmação visto que ainda existe um longo caminho e ser percorrido pela reforma psiquiátrica -, de maneira a manter acesa a chama da luta antimanicomial, na busca de uma constante evolução e no combate aos retrocessos. Amarante (2003) destaca também que os CAPS devem ser um serviço inovador.

Segundo Rosa (2016):

Esses serviços precisam ser espaços de criação de novas
práticas sociais, de novos conceitos e de novas formas de
vida. Garantir aos trabalhadores dos CAPS a consciência
de que devem, com suas práticas, produzir rupturas
conceituais, técnicas, políticas, jurídicas e sociais é um
desafio do ponto de vista da avaliação qualitativa da
política de saúde mental no que diz respeito a ampliação
da cobertura de serviços CAPS. (ROSA, 2016, p. 119)

Dessa forma, é possível também questionar o papel dos trabalhadores em saúde mental na efetivação de tais políticas e na difusão de novas e melhores práticas. Ainda existe uma luta política e um investimento ético? 


\section{O papel dos CAPS no contexto pós reforma:}

Segundo Silva e Silva (2009), a partir de 2001, com a aprovação da Lei $n^{\circ} 10.216$, ocorreram mudanças significativas para a consolidação dos direitos dos portadores de doenças mentais. As autoras destacam a orientação do Ministério da Saúde, sendo esta:

Repensar o modelo de atendimento; criando serviços alternativos as instituições asilares, como os Centros de Atenção Psicossocial (CAPS), hospital dia, residências terapêuticas e leitos em hospitais gerais, que se propõem a permitir que pessoas com problemas psiquiátricos não sejam excluídas do convívio social. (SILVA e SILVA, 2009, p. 1)

De acordo com o Ministério da Saúde (BRASIL, 2015), os CAPS fazem parte das Redes de Atenção Psicossocial (RAPS) e se caracterizam como um serviço de saúde de caráter aberto e comunitário, formado por uma equipe multiprofissional interdisciplinar com a funçãode realizar atendimento às pessoas com sofrimento ou transtorno mental, além de também necessidades decorrentes do uso de álcool e drogas, sendo substituto do modelo asilar. Os mesmos operam em territórios, que se configuram não apenas geograficamente, mas também como territórios pessoais, onde se desenvolvem a vida dos usuários e suas famílias, sendo então, um lugar referenciado como provedor de cuidado e vida, com a missão de garantir a cidadania e a inclusão dos usuários e familiares (BRASIL, 2015).

Pode-se destacar então o papel dos CAPS dentro do Sistema Único de Saúde (SUS), para o Ministério da Saúde (BRASIL, 2004), o objetivo dos CAPS é:

[...]oferecer atendimento à população de sua área de abrangência, realizando o acompanhamento clínico e a reinserção social dos usuários pelo acesso ao trabalho, lazer, exercício dos direitos civis e fortalecimento dos laços familiares e comunitários. (BRASIL, 2004, p. 13)

Dentro das RAPS, os CAPS visam a possibilidade de vida comunitária e a autonomia dos usuários, através de um trabalho em equipe com o objetivo de buscar recursos em outras redes, assim como nos territórios.

O cuidado promovido pelo CAPS ocorre através do Projeto Terapêutico Singular (PTS), que envolve a equipe, o usuário e seus familiares em sua elaboração, tendo o CAPS a responsabilidade da ordenação do cuidado.

As práticas ocorremem ambientes "portas abertas" - no sentido literal e simbólico - dentro do território do usuário, onde são projetados serviços públicos de saúde -tais como a prestação de atendimento em regime diário, inserção dos usuários em ações intersetoriais, atendimento individual ou grupal, atendimento para familiares, entre outros -, sendo substitutivos do modelo asilar, e funcionando como referência nos territórios. Dessa forma, é um:

"[...] espaço de cuidar e apoiar pessoas com experiência do sofrimento e, ao mesmo tempo, espaço social no sentido de produção de projetos de vida e de exercício de direitos, e de ampliação do poder de contratualidade social. " (BRASIL, 2015. p. 24)

Os CAPS devem possuir um espaço próprio e adequado a sua demanda. Ainda segundo o Ministério da Saúde (BRASIL, 2004),

As práticas realizadas nos CAPS se caracterizam por ocorrerem em ambiente aberto, acolhedor e inserido na cidade, no bairro. Os projetos desses serviços, muitas vezes, ultrapassam a própria estrutura física, em busca da rede de suporte social, potencializadora de suas ações, preocupando-se com o sujeito e sua singularidade, sua história, sua cultura e sua vida quotidiana. [...] esses recursos vão além do uso de consultas e de medicamentos, e caracterizam o que vem sendo denominado clínica ampliada. Essa ideia de clínica vem sendo (re)construída nas práticas de atenção psicossocial, provocando mudanças nas formas tradicionais de compreensão e de tratamento dos transtornos mentais. (BRASIL, 2004, p. 14)

Contudo, de acordo com Ballarin et al (2011 apud QUEIROZ et al, 2013, p.128) mesmo com o grande avanço na atenção à saúde mental, a assistência integral e mais humanizada ainda é uma prática a ser atingida, visto que é possível perceber no cotidiano dos serviços atuações fragmentadas e desarticuladas, advindas principalmente a existência de duas lógicas opostas, sendo uma voltada ao modelo médico, com práticas centradas na pessoa, e outra visando um cuidado integral em rede.

Segundo Oliveira (2000):

\begin{abstract}
Criar novos serviços de cuidado da saúde mental não significa a transformação da lógica manicomial e da forma de compreender e praticar a psiquiatria. Assim, é preciso cuidado para não correr o risco de se desenvolver práticas antigas com uma nova roupagem, seja por falta de conhecimento por parte dos profissionais, seja pela filosofia de serviço. (OLIVEIRA apud QUEIROZ et al, 2013, p. 135)
\end{abstract}

O movimento antimanicomial possui uma grande força, e desde seu início, já foram obtidos avanços significativos, sendo importante, para que tais avanços continuem a ocorrer, o engajamento dos profissionais em saúde mental na problematização dos possíveis retrocessos e rupturas, assim como movimentos políticos que possam levar a um progressivo desmonte da reforma, a partir de retrocessos anunciados, tais como a nota técnica $11 / 2019$, que foi inclusive alvo de repúdio por parte do Conselho Federal de Psicologia (CFP). Esta nota técnica apresenta "esclarecimentos sobre as mudanças na política nacional de saúde mental e nas diretrizes 
da política nacional sobre drogas" e foi repudiada por conter propostas incoerentes com os avanços obtidos. Assim, é preciso sempre haver um questionamento acerca das práticas, em busca da existência de novas formas de institucionalização a serem combatidas, como por exemplo a medicalização, que será discutida nos próximos tópicos. Tais questionamentos devem ocorrer com base no que foi proposto em comparação ao que tem ocorrido no panorama atual, sempre reafirmando o papel ético e de combate, característico da reforma e da luta antimanicomial, em busca de um cuidado humanizado e pautado na garantia de direitos.

\section{A medicalização como meio de controle social:}

A medicalização consiste no uso de fármacos como meio terapêutico sem escuta clínica. Acerca de sua utilização, é necessário considerar que, atualmente, a relação entre a psiquiatria e a indústria farmacêutica tem se tornado clara, gerando assim questionamentos sobre a eficácia e base para o emprego desta prática. Apesar da reforma psiquiátrica não ter focado nesta temática, Barros e Yasui (2017), em seu trabalho, apresentam uma crítica pela atual difusão da prática e apontam estudos anteriores que demonstram a medicalização como uma prática não reformada em saúde mental.

Assim, apesar dos avanços já obtidos, ainda são necessárias muitas mudanças nas práticas de atenção, entre as quais, é apontada por Onocko-Campos (2013) a primazia do tratamento farmacológico, sendo por diversas vezes, o único tratamento proposto.

A medicalização leva a uma ampliação do exercício da medicina sobre a sociedade, dessa forma, pode-se dizer que o uso de psicofármacos se configura também como uma nova forma de institucionalização, pois apesar de os indivíduos não estarem mais internados e possuírem certa autonomia, esta autonomia é controlada através dos medicamentos, funcionando como um meio de controle social.

Ao se falar em medicalização, é importante ressaltar também que, atualmente, o número de transtornos mentais conhecidos é muito maior do que há 60 anos, porém, mesmo que se considere a evolução científica, segundo Onocko-Campos (2013):

Não se pode ignorar que é justamente a indústria farmacêutica quem financia grande parte das pesquisas e eventos educativos na área, consolidando-se como uma das indústrias mais lucrativas no mundo e influenciando a produção científica. (ONOCKO-CAMPOS, 2013, p. 2890)

Também é apontada por Onocko-Campos (2013) a prática da hospitalização e renovação de receitas sem consultas como resposta comum às demandas, se tornando parte do problema, pois, segundo o autor, os indivíduos passam a utilizar medicamentos por tempo indeterminado e são submetidos a um cuidado fragmentado.

Onocko-Campos (2013) destaca como desafio:

Estimularaautonomiaeopoderdeagirdosusuáriosemobilizálos para que descubram ou redescubram seus interesses e desejos, [...] um cuidado cujas principais preocupações deixem de ser o diagnóstico, a doença e a prescrição medicamentosa. (ONOCKO-CAMPOS, 2013, p. 2897)

Costa-Rosa (2013), cita também que o consumo de psicofármacos é um sintoma social, ao comparar sua utilização com sua real necessidade, assim, o autor deixa claro que se deve buscar a diminuição do uso "compulsório", e não necessariamente sua abolição.

\section{A necessidade de mudança no pensamento da sociedade:}

Para Dimenstein e Liberato (2009), a reforma levou uma maior visibilidade a produção de novos modos de subjetivação, propondo práticas novas que rompem com a lógica tutelar.

A partir da doença mental, surgem práticas de controle, tutela, medicalização, etc., que são evidentes no cotidiano. Segundo Machado e Lavrador (2001):

\begin{abstract}
Queremos mudar, mas esse querer vai sendo enfraquecido, pois também está atravessado por uma lógica, aqui entendida como marcas invisíveis que produzem formas de subjetivação, que se expressa através de um desejo em nós de dominar, de subjugar, de classificar, de hierarquizar, de oprimir e de controlar a vida. (MACHADO E LAVRADOR 2001, apud DIMENSTEIN e LIBERATO, 2009, p. 1)
\end{abstract}

Dessa forma, pode-se dizer que a reforma psiquiátrica possui âmbito subjetivo, pois é atravessada por conceitos enraizados, necessitando que haja uma mudança de pensamento na sociedade, de forma a ampliar sua força.

É possível perceber grandes avanços na reforma psiquiátrica e um quadro promissor de contínuo desenvolvimento, porém também, inúmeros desafios.

Segundo Dimenstein e Liberato (2009) o maior indicador do avanço da reforma, é o aumento do número de CAPS, porém, por não haver o preparo adequado, apontam que os mesmos indicam também a produção de novas ordens de cronicidade.

Sobre as fragilidades da reforma, Dimenstein e Liberato (2009) afirmam:

Se a constituição de uma rede de serviços substitutivos integrada entre si e com outros equipamentos sociais presentes nas comunidades é algo imprescindível para o avanço da reforma, esse é um dos aspectos que apresentam mais fragilidades, pois, de fato, ainda não dispomos de uma rede ágil, flexível, resolutiva, onde o transito dos usuários é facilitado e o mesmo acolhido em suas diferentes 
demandas. (DIMENSTEIN; LIBERATO, 2009, p. 5)

Além de problemas institucionais, outro desafio apontado por Dimenstein e Liberato (2009) é a vontade de alguns pacientes de permanecerem sob tutela, por questões de segurança, condições básicas de sobrevivência e tratamento de saúde.

Lutamos por reinserção, reabilitação e cidadania para portadores de transtornos mentais quando essa questão se refere ao fato de que a constituição da cidadania em um país como o Brasil é menos uma questão relacionada a condição de louco, e mais uma questão social, tendo em vista a grande parcela da população brasileira que não desfruta das condições de vida identificadas como cidadania. (DIMENSTEIN; LIBERATO, 2009, p. 6)

Dessa forma, a reforma fica restrita aos serviços de saúde, sem a ampliação de redes assistenciais, que se torna limitada e proporciona poucas possibilidades de reinserção social.

Dimenstein e Liberato (2009) apresentam como consequência da cronificação provocada pela falta de articulação entre os serviços e apoios sociais, a burocratização das práticas e o formato das redes.

Adesinstitucionalizaçãoédefinida por Dimenstein e Liberato (2009, p.9) como "ultrapassar fronteiras sanitárias, é enfrentar o desafio da intersetorialidade e do trabalho em rede, o que implica na adoção de modelos de atenção integral de base territorial."

Como solução proposta, os autores citam o investimento em uma rede diversificada de dispositivos de auxílio ao usuário e sua família, ajudando a enfrentar momentos de crise e fortalecendo a atenção básica.

\section{O panorama atual}

Inicialmente, é importante sinalizar que uma nota técnica é um documento de análise sobre uma política pública ou programa de governo, com intuito de avaliar seu funcionamento e buscar alternativas para superar dificuldades.

No dia 04 de fevereiro de 2019 foi lançada pelo Ministério da Saúde, a nota técnica "Nova Saúde Mental" $n^{\circ} 11 / 19$, na qual são discutidos os rumos da saúde mental, com propostas que vão contra às resoluções da lei da Reforma Psiquiátrica (Lei $\left.n^{\circ} 10.216 / 2001\right)$. Neste contexto, pode-se afirmar que o atual panorama exige cautela e, mais do que nunca, a resistência e apoio por parte dos profissionais em saúde mental, de forma a evitar o desmonte da reforma e os possíveis retrocessos anunciados.

Uma das grandes modificações observadas na nota técnica é o fato de a mesma recolocar os hospitais psiquiátricos como um serviço a ser fortalecido, não incentivando mais seu fechamento gradual.

Em 1987, se iniciava a luta antimanicomial, após a formulação e assinatura da carta de Bauru por parte dos trabalhadores em saúde mental, onde o grande fator a ser combatido era a estigmatização da loucura, buscando a garantia de direitos e o acesso a um melhor cuidado. A partir de então, foi criada a RAPS, sendo chamada de rede substitutiva e oferecendo serviços desenvolvidos como alternativas ao modelo asilar, na busca de um cuidado humanizado, com garantia de direitos e vivencia familiar nos próprios territórios, onde os indivíduos pudessem conviver em sociedade.

Ainda assim, a partir do exposto acima, vemos que os objetivos propostos pela reforma ainda não foram totalmente concretizados, porém, é necessário também reconhecer que a partir da reforma psiquiátrica, o cuidado em saúde mental já avançou significativamente, portanto, deve-se combater retrocessos, assim como sinalizou o Conselho Federal de Psicologia (CFP), ao lançar uma nota de repúdio à nota técnica do Ministério da Saúde.

De acordo com o CFP:

[...] a nota apresenta, entre outras questões que desconstroem a política de saúde mental, a indicação de ampliação de leitos em hospitais psiquiátricos e comunidades terapêuticas, dentro da rede de atenção psicossocial (RAPS), incentivando assim o retorno à lógica manicomial. O Ministério da Saúde também passa a financiar a compra de aparelhos de eletroconvulsoterapia. (CFP, 2019)

A partir dos dados já expostos, pode-se realizar um comparativo entre as diretrizes propostas pela lei da Reforma Psiquiátrica (Lei ${ }^{\circ}{ }^{10.216 / 2001)}$ e a nota técnica.

\section{O que muda?}

Em 1989, se iniciou o trâmite acerca da lei da Reforma Psiquiátrica, através do Projeto de Lei (PL) 3657/1989, do deputado Paulo Delgado. A partir de então, houve um longo caminho até a promulgação da lei $\mathrm{n}^{\circ} 10.216$, de 6 de abril de 2001.

O PL proposto visava a regulamentação dos direitos das pessoas com transtornos mentais e a extinção progressiva dos manicômios do país, através de sua substituição por outros recursos assistenciais, além de regulamentar a internação compulsória.

Porém, como citado, foi somente em 2001, após 12 anos de tramitação e debates no Congresso Nacional, que a Lei Federal $\mathrm{n}^{\circ} 10.216$ foi sancionada pelo então presidente, Fernando Henrique Cardoso. No entanto, a lei aprovada é uma substituição do projeto de lei inicial, apresentando modificações no texto. Sendo assim, a lei 10.216 "dispõe sobre a proteção e os direitos das pessoas portadoras de transtornos mentais e redireciona o modelo assistencial em saúde mental".

$\mathrm{Na}$ lei, entre os direitos da pessoa portadora de transtorno mental, encontram-se os seguintes: "ser 
tratada com humanidade e respeito e no interesse exclusivo de beneficiar sua saúde, visando alcançar sua recuperação pela inserção na família, no trabalho e na comunidade", além de "ser tratada em ambiente terapêutico pelos meios menos invasivos possíveis". (BRASIL, 2001)

$\mathrm{O}$ artigo 4 da referida lei, sinaliza que a indicação para internação ocorrerá somente quando os recursos extra hospitalares se mostrarem insuficientes, tendo como finalidade de tratamento, a reinserção social do paciente no seu meio.

Os parágrafos 2 e 3 , ainda no artigo 4 , tratam sobre a estrutura do regime de internação, que deverá oferecer assistência integral à pessoa portadora de transtorno mental, incluindo serviços médicos, de assistência social, psicológico, ocupacionais, de lazer e outros, sendo vetada a internação em instituições com características asilares, ou seja, desprovida dos recursos mencionados.

Acerca das políticas de reabilitação social, no artigo 5, é dito que para pacientes institucionalizados há longo tempo ou em situação de grave dependência, este será objeto de política específica de alta planejada e reabilitação psicossocial assistida.

Sendo assim, observa-se alguns aspectos da lei da reforma psiquiátrica, os quais se apresentam contra as internações com características asilares, tendo como finalidade de tratamento a reinserção do paciente na sociedade e a garantia de seus direitos.

Agora serão apresentadas as mudanças propostas pela nota técnica 11/2019.

A nota técnica 11/2019, segundo seu texto, promove mudanças na Política Nacional de Saúde Mental, com o objetivo de torná-la mais acessível, eficaz, resolutiva e humanizada. Além de afirmar que tal iniciativa enfrenta desafios e problemas já conhecidos, porém, mantendo a essência e respeito à Lei 10.216/2001.

Ainda segundo o texto:

O objetivo é fazer com que os pacientes, dos casos mais complexos aos mais graves, tenham acesso a tratamento efetivo no SUS, de acordo com suas necessidades e peculiaridades, sem deixar de lado nenhuma modalidade de tratamento validada e aplicável. (BRASIL, 2019, p. 3.)

A seguir, o texto fala sobre as RAPS, apresentando sua nova composição, que passa a incluir os hospitais psiquiátricos, afirmando que todos os serviços "são igualmente importantes e devem ser incentivados, ampliados e fortalecidos" (BRASIL, 2019).

Indica também que não há mais porque se falar em "rede substitutiva", visto que não se considera mais os serviços como substituíveis, atribuindo a cada um seu papel, e não mais incentivando o fechamento de unidades de qualquer natureza.

Também é apontado como problema, o número de leitos psiquiátricos existentes atualmente, que passará a ser expandido. Além disso, afirma que os hospitais psiquiátricos devem ser modernizados e seguir protocolos e padrões modernos vigentes, mantendo o incentivo a desinstitucionalização, pois não entende tais hospitais como local de moradia, porém, tal desinstitucionalização não será mais sinônimo de fechamento de leitos e de hospitais psiquiátricos.

Pode-se observar em um trecho do texto, a afirmação de que o baixo número atual de leitos existentes "é fruto direto dos equívocos de fechamento de leitos psiquiátricos no Brasil, nas duas últimas décadas, principalmente" (BRASIL, 2019), onde é realizada uma crítica à reforma psiquiátrica, que pode ser refutada a partir dos dados expostos e das discussões realizadas, pois, juntamente com a diminuição dos leitos e o fechamento dos hospitais psiquiátricos, foi proposta a criação e efetivação de uma rede substitutiva que viesse a dar conta dessas questões de maneira humanizada e com garantias de direitos.

Ainda segundo o documento, foram encontrados erros na rede substitutiva, tais como: incentivos financeiros não utilizados, subnotificação de atendimentos, denúncias de violações de direitos, etc. Sendo assim, porque não realizar uma maior fiscalização, mantendo os moldes atuais?

Outra parte preocupante, é a questão do tratamento oferecido aos pacientes, como por exemplo a eletroconvulsoterapia (ECT), cujo aparelho agora passa a ter sua compra financiada pelo Ministério da Saúde.

A eletroconvulsoterapia é um procedimento que consiste na indução de crises convulsivas por meio da passagem de uma corrente elétrica pelo cérebro com fins terapêuticos (Antunes et al, 2009). Seu tratamento, também chamado de "eletrochoque" era frequente nos hospitais psiquiátricos e é relatado por Arbex, no livro "Holocausto Brasileiro" (2013), segundo a autora:

A eletroconvulsoterapia existe, desde 1938, para tratamento de doenças mentais, mas seu uso, no século passado, foi muito controverso. A tecnologia do eletrochoque se modernizou há um par de décadas, sendo utilizada nos dias atuais com fins terapêuticos para alguns tipos de transtornos, como a depressão profunda, embora existam correntes contrárias ao seu uso. No Brasil, o método só passou a ter mais controle em 2002, quando o Conselho Federal de Medicina estabeleceu regras específicas para a adoção da técnica, como a necessidade de aplicar anestesia geral. Além da anestesia, a utilização de relaxantes musculares ameniza as convulsões, mas nem sempre foi assim. No Colônia, o choque era aplicado a seco e tinha características semelhantes à tortura. (ARBEX, 2013, p. 32.

Ainda de acordo com Arbex (2013), o eletrochoque era aplicado indiscriminadamente, e, por vezes, a energia elétrica da cidade não era suficiente para aguentar a carga. Dessa forma, por seu uso indiscriminado, o eletrochoque causou diversas mortes 
dentro dos hospitais psiquiátricos.

Entre outras questões, destacaram-se como principais, a volta dos hospitais psiquiátricos e a compra de eletroconvulsores, dessa forma, pode-se afirmar, ainda de acordo com o CFP, que tais medidas desconstroem a política de saúde mental, sendo importantes pontos de retrocessos à luta antimanicomial. Portanto, no panorama atual, é preciso lutar contra tais retrocessos, sendo iniciado um novo marco na luta antimanicomial.

\section{Considerações Finais}

Pode-se afirmar o caráter permanente da reforma psiquiátrica, através de diversos caminhos ainda a serem traçados, assim, o artigo buscou contribuir ao meio acadêmico como um norteador de práticas ainda a serem reformadas, de maneira a estimular novos estudos, e também apresentar o panorama atual.

No artigo, procurou-se apresentar tópicos presentes nos objetivos da reforma e realizar uma comparação com as práticas atuais, dessa forma, foi demonstrado que ainda existem áreas não reformadas e também novas formas de institucionalização.

Viu-se que o objetivo da reforma não era apenas a criação de CAPS, e sim a criação de uma rede de apoio completa, buscando um melhor atendimento para pacientes com transtornos mentais. Sendo assim, é possível pensar se realmente há possibilidade de reinserção social nos moldes atuais da reforma, visto que apenas a implementação dos CAPS não abrange o objetivo geral proposto, pois vemos um cuidado fragmentado, muitas vezes devido à grande demanda.

Dessa forma, viu-se que, apesar dos resultados já alcançados, ainda há muito a ser feito, principalmente no que tange ao combate a retrocessos, nos servindo como forma de resistência, através da continuidade dos princípios que norteiam nossa práxis, mantendo nosso papel na promoção dos Direitos Humanos, da inclusão e da garantia de direitos.

É possível também constatar que mesmo com os avanços notórios obtidos pela reforma, existem sim novas formas de institucionalização, além de práticas a serem repensadas com intuito de chegar ao real objetivo da reforma, sendo também importante reconhecer que a partir da reforma, o cuidado em saúde mental evoluiu de maneira significativa.

Assim, espera-se que os levantamentos apresentados acerca de tais desafios possam vir a contribuir para um maior avanço da reforma, buscando uma real inserção à sociedade e novas e melhores formas de lidar com tais pacientes.

\section{Referências:}

AMARANTE, P. A (clínica) e a Reforma Psiquiátrica. In Archivos de Saúde Mental e Atenção Psicossocial, p. 45-65. Rio de Janeiro: Editora NAU,
2003.

ANTUNES, P. B., et al. Eletroconvulsoterapia na depressão maior: aspectos atuais. Rev. Bras. Psiquiatr.,São Paulo, v. 31, supl .1. p. S26s33. 2009. Disponível em: <http://www.scielo.br/scielo.php?pid=S1516$44462009000500005 \&$ script $=$ sci abstract\&tlng=pt $>$. Acesso em 14 abr 2019.

ARBEX, D. Holocausto Brasileiro. São Paulo: Geração Editorial, 2013.

BARROS, A.C.F.; YASUI, S. Medicalização e Atenção Psicossocial: um encontro arriscado. $3^{\circ}$ Fórum de direitos humanos e saúde mental. Universidade Federal de Santa Catarina, 2017.

BIRMAN, J; COSTA, J. F. Organização de instituições para uma psiquiatria comunitária. Psiquiatria social e reforma psiquiátrica. Rio de Janeiro: FIOCRUZ; 1994. p. 41-72

BRASIL, Ministério da Saúde. Lei n. 10.216, de 6 de abril de 2001. Dispõe sobre a proteção e os direitos das pessoas portadoras de transtornos mentais e redireciona o modelo assistencial em saúde mental. Diário Oficial da União, seção 1,2001. Disponível em: <http://www.planalto.gov.br/ccivil 03/leis/ LEIS 2001/L10216.htm>. Acesso em 03 abr2019.

BRASIL, Ministério da Saúde. Saúde mental no SUS: os centros de atenção psicossocial. Brasília: Ministério da Saúde, 2004.

BRASIL, Ministério da Saúde. Centros de Atenção Psicossocial e Unidades de Acolhimento como lugares da atenção psicossocial nos territórios: orientações para elaboração de projetos de construção, reforma e ampliação de CAPS e de UA. Brasília: Ministério da Saúde, 2015. Disponível em: $<$ http://bvsms.saude.gov.br/bvs/publicacoes/centros atencao_psicossocial unidades acolhimento.pdf $>$. Acesso em: 14 nov 2018

BRASIL, Ministério da Saúde. Nota Técnica $n^{\circ} 11 / 2019$. Esclarecimentos sobre as mudanças na Política Nacional de Saúde Mental e nas Diretrizes da Política Nacional Sobre Drogas. Ministério da Saúde, 2019. Disponível em: $<$ http://pbpd.org.br/wp-content/uploads/2019/02/0656ad6e.pdf $>$. Acesso em 04 abr 2019.

BUENO, M.L.S.; CAPONI, S. A construção do discurso dos sujeitos envolvidos com o processo de reforma psiquiátrica: um estudo sobre o município de Joinville/SC. Interface - Comunicação, Saúde, Educação, v.13, n.28, p.137-50, jan/mar. 2009. Disponível em: <http://www.scielo.br/pdf/ icse/v13n28/v13n28a12.pdf>. Acesso em 03 abr 2019.

CONSELHO FEDERAL DE PSICOLOGIA. Nota de Repúdio a Nota Técnica "Nova Saúde Mental". Conselho Federal de Psicologia, 2019. Disponível em: <https://site.cfp.org.br/cfp-manifesta-repudio-a-notatecnica-nova-saude-mental-publicada-pelo-ministerio-da-saude/>. Acesso em 03 abr 2019.

COSTA-ROSA, A. Atenção Psicossocial além da Reforma Psiquiátrica: contribuições a uma Clínica Crítica dos processos de subjetivação na Saúde Coletiva. São Paulo: Editora Unesp, 2013.

DIMENSTEIN, M.; LIBERATO, M. Desinstitucionalizar é ultrapassar fronteiras sanitárias: o desafio da intersetorialidade e do trabalho em rede. Cad. Bras. Saúde Mental, v.1, n.1, p.1-10, 2009

MACEDO, J.P.; DIMENSTEIN, M. Ação Política-Profissional dos Psicólogos e a Reforma Psiquiátrica. Estudos de Psicologia, Natal, v. 18, n. 2, p. 297-304, 2013. Disponível em: <http://www.scielo.br/pdf/epsic/v18n2/ v18n2a15.pdf>. Acesso em 03 abr 2019.

ONOCKO-CAMPOS, R. T. et al. A Gestão Autônoma da Medicação: uma intervenção analisadora de serviços em saúde mental. Ciênc. Saúde coletiva, Rio de Janeiro, v. 18, n. 10, p. 2889-2898. 2013. Disponível em: $\quad<$ http://www.scielo.br/scielo.php?script=sci arttext\&pid=S1413 $81232013001000013 \& \operatorname{lng}=$ en\&nrm=iso $>$. Acesso em 05 set 2018

QUEIROZ, Y. L. et al. Práticas em um CAPS de minas gerais: o relato de uma experiência. Rev. SPAGESP, Ribeirão Preto, v. 14, n. 2, p. 126-137, 2013.Disponível em: <http://pepsic.bvsalud.org/scielo.php?script=sci arttext\&pid $=$ S1677-29702013000200010\&lng=pt\&nrm=iso $>$. Acesso em 08 nov 2018

ROSA, E. Z. Por uma Reforma Psiquiátrica Antimanicomial: o papel estratégico da Atenção Básica para um projeto de transformação social. 2016. 380 f. Tese (Doutorado em Psicologia: Psicologia Social) - Programa de Estudos Pós-Graduados em Psicologia: Psicologia Social, Pontifícia Universidade Católica de São Paulo, São Paulo, 2016. 
SILVA, M. D.; SILVA, R. C. O Benefício da Prestação Continuada e o transtorno mental: uma análise dos impactos na vida dos usuários do CAPS II do Município de Campina Grande. XIII Encontro Latino Americano de Iniciação científica e IX Encontro Latino Americano de pós-graduação. Universidade do Vale do Paraíba, 2009.

TENORIO,F.Areformapsiquiátricabrasileira, dadécadade 1980aosdiasatuais: história e conceitos. Hist. cienc. Saúde-Manguinhos, Rio de Janeiro, v. 9, n. 1, p. 25-59. 2002. Disponível em $<$ http://www.scielo.br/scielo.php?script=sci arttext\&pid $=$ S0104-59702002000100003\&lng=en\&nrm=iso>. Acesso em 05 set 2018.

VENTURINI, E. A desinstitucionalização: limites e possibilidades. Rev. bras. Crescimentodesenvolv. hum. São Paulo, v. 20, n. 1, p. 138-151. 2010. Disponível em $<$ http://pepsic.bvsalud.org/scielo.php?script=sci arttext\&pid $=$ S0104-12822010000100018\&lng $=$ pt\&nrm=iso $>$. Acesso em 08 nov 2018. 